\title{
Self-directed learning: Status of final-year students and perceptions of selected faculty leadership in a Nigerian medical school - a mixed
} analysis study

\author{
T E Nottidge, ${ }^{1}$ MBBS, MPhil Health ScEd, FWACS; A J N Louw, ${ }^{2}$ BEd, MEd, PhD \\ ${ }^{1}$ Department of Orthopaedics and Traumatology, College of Health Sciences, University of Uyo, Nigeria \\ ${ }^{2}$ Centre for Health Professions Education, Faculty of Medicine and Health Sciences, Stellenbosch University, Cape Town, South Africa
}

Corresponding author: T E Nottidge (timnottidge@yahoo.com)

\begin{abstract}
Background. Self-directed learning (SDL) is the essential mechanism of lifelong learning, which, in turn, is required for medical professionals to maintain competency because of advancing technology and constantly evolving disease care and contexts. Yet, most Nigerian medical schools do not actively promote SDL skills for medical students.

Objective. To evaluate the status of SDL behaviour among final-year students, and the perceptions of faculty leadership towards SDL in a Nigerian medical school Methods. A mixed research method was used, with a survey consisting of a validated Likert-based self-rating scale for SDL (SRSSDL) to assess students SDL behaviour. Focus group discussions with selected faculty leaders were thematically analysed to assess their perceptions of SDL.

Results. The medical students reported moderate SDL behaviour, contrary to faculty, who considered their students' SDL behaviour to be low. Faculty leadership further defined SDL as the self-motivated student demonstrating initiative in learning under the guidance of teachers, who use interactive forums for teaching. Furthermore, teachers and students should partner towards the goal of ensuring that student learning takes place. Teachers expressed concerns about SDL methods in medical schools owing to the fear that this will require medical students to teach themselves medicine without expert guidance from teachers.

Conclusion. This study suggests that final-year students have a low to moderate level of SDL behaviour. The index faculty are willing to develop teacherguided self-motivated learning for their students, rather than strict SDL. Faculty should be concerned about this behaviour and should encourage SDL in such a way that students realise its benefits to become lifelong learners. Further study of the perceptions about self-regulated learning are recommended
\end{abstract}

Afr J Health Professions Educ 2017;9(1):29-33. DOI:10.7196/AJHPE.2017.v9i1.708

Knowles $^{[1]}$ defined self-directed learning (SDL) as 'a process in which individuals take the initiative, with or without the help of others, in diagnosing their learning needs, formulating learning goals, identifying human and material resources for learning, choosing and implementing appropriate learning strategies and evaluating learning outcomes'. This definition is difficult to apply in an institutional setting, ${ }^{[2]}$ and more so in the complex process of imparting the defined knowledge, skills and attitudes required to produce a medical doctor. In formal education, self-regulated learning (SRL) is a more feasible framework of a student's proactive approach to learning. ${ }^{[3]}$

SRL 'describes the proactive, self-directive processes and self-beliefs, by which students become masters of their own learning. ${ }^{[4]}$ Developing skills in SRL requires the student's 'personal initiative, perseverance and adaptive skill that derives from advantageous motivational feelings and beliefs and metacognitive strategies. ${ }^{\text {'[] }}$ The three skills that comprise SRL are metacognition, motivation and cognition. ${ }^{[5]}$ SRL expresses a student's motivation to achieve defined academic goals utilising specific strategies, which leverage on self-efficacy beliefs. ${ }^{[4]}$ Therefore, the concepts of being proactive, motivated to learn, and teacher guidance are defined components of SRL. ${ }^{[5]}$ The teacher can leverage the cognitive load theory to create a conducive atmosphere for learning.

The key concept of the cognitive load theory is that the cognitive load should match the working memory of the learner. Cognitive load occurs when several new facts (sensory information) are received without scaffolding. ${ }^{[6]}$ Teacher support is needed to provide the scaffolding to help the student 'make sense' of the new information. ${ }^{[7]}$ This teacher-directed learning has contextual overtones in the hierarchical culture of many higher education institutions, especially in Africa.

SDL in a problem-based learning (PBL) curriculum in developing countries reveals difficulties with its implementation owing to high start-up costs and the need for well-trained facilitators. This has been observed, for example, in Argentina, South-East Asia and sub-Saharan Africa (mainly in South Africa). ${ }^{[8-10]}$ In the West African sub-region and specifically in Nigeria, PBL is yet to be established, although the College of Medicine, University of Ibadan, Nigeria has started implementing this programme. ${ }^{[11-13]}$ Therefore, it would seem that most learning in Nigerian medical schools is not student centred or focused on developing lifelong learning skills. These skills would enhance a professional's relevance after formal education and thus promote safe, efficient medical care. However, published work on structured medical education in Nigeria is scanty; information on types of curricula is mostly not available in public repositories.

This study attempts to answer the following question: What is the current state of SDL among the students (as trainees) and its perception among the faculty leadership (as trainers) at a Nigerian medical school? It appears to be the first structured assessment of SDL status and perception in a Nigerian medical school - its findings are transferable to other medical schools in 
the developing world, which are yet to develop SDL and lifelong learning in their curricula.

\section{Methods}

A sequential mixed method approach - first quantitative and then qualitative methods - was used to answer the research question of this study. ${ }^{[14]}$ The quantitative data formed part of the material for discussion in the qualitative arm of the study. It therefore follows an explanatory design, where the qualitative findings help to clarify the quantitative findings. Therefore, the research followed separate phases - first the quantitative phase, followed by the qualitative phase. A third phase of the study involved reviewing the findings from the focus group discussion with the members of the group on an individual basis, thus providing data triangulation and further enriching the data. ${ }^{[14]}$

The phases of the study were as follows:

- Phase I (quantitative phase): self-rating scale for SDL (SRSSDL) administered to the final-year students of the index medical school.

- Phase II (qualitative phase): focus group discussion.

- Phase III: member-checking phase; results of phases I and II discussed with members of the focus group individually. This was done to enhance the validity and trustworthiness of the data.

The SRSSDL was designed by Williamson ${ }^{[15]}$ to assess SDL behaviour - the user's level of self-directedness in learning is graded on an ordinal scale as low, medium or high. The SRSSDL is a paper questionnaire that has 60 positively worded questions, with answer options on a Likert scale. The question domains are: (self) awareness, learning strategies, learning activities, (self) evaluation and interpersonal skills. A scoring rubric is embedded after the questions, which allows for self-scoring, although the self-scoring option was not used in this study.

Low SDL is defined in the SRSSDL as 'guidance is definitely needed from the teacher. Any specific changes necessary for improvement must be identified and a possible complete re-structuring of the methods of learning.' In addition, medium SDL is defined as 'this is half-way to becoming a self-directed learner. Areas for improvement must be identified, evaluated and a strategy adopted with teacher guidance when necessary.' High SDL 'indicates effective self-directed learning. The goal now is to maintain progress by identifying strengths and methods for consolidation of the students' effective self-directed learning.'

Purposive sampling was used for this study. The choice of faculty was based on the criteria that those chosen would be a head of department, considered likely to attend the event, and also the two deans involved. Nine staff members were chosen -3 of 3 for the basic sciences, 4 of 13 for the clinical sciences, and the 2 deans - all those selected attended or sent a representative for the focus group discussion. The selection of deans and heads of department ensured that the opinion of faculty leadership was engaged, because they play a central role in managing the teaching and learning policies of the medical school.

The questionnaires completed during phase I were collected and graded and the scores were captured on a Microsoft Excel (USA) spreadsheet for analysis. During phase II, the focus group discussion was recorded on both audio and video devices and a manual transcript of the entire audio recording was made. The feedback obtained in phase III was worked into and enriched the data analysed in phase II.

Non-crossover mixed analysis was used to analyse the data. The quantitative data were captured on a Microsoft Excel spreadsheet and basic descriptive statistical data of the students' ages and scores were obtained - frequencies and means, respectively. The overall mean score was used to classify the group into low, medium or high SDL behaviour, using the scoring rubric embedded in the SRSSDL.

Ethical approval for this study was obtained from the ethics committee of the Faculty of Medicine and Health Sciences, Stellenbosch University (ref. no. S14/02/033). Permission to carry out the study for the purpose of college development and curriculum review was obtained from the chief executive officer of the medical school. The study was carried out in 2014 .

\section{Results}

Following an explanatory design for the mixed method study, the results are presented accordingly - the quantitative findings are followed by the results of the qualitative phase of the study.

\section{Students' self-reported SDL behaviour}

Forty of 43 final-year students participated in this study - a 93\% response rate. The 3 students who did not participate were not available at the time. Ten of the participants were female and 30 male; 34 were in the 20 -29-year age group, while 6 were in the 30 - 39-year age group.

The mean (standard deviation) for self-directed behaviour was 212.3 (21.2) (Fig. 1), which is within the medium range of the SRSSDL scale.

\section{Faculty's perception of SDL}

Table 1 is a summary of the results of the qualitative aspect of the focus group discussion.

\section{Positive concepts}

At the start of the focus group discussion, faculty observed that the term SDL was new to them. In this first part of the discussion, faculty considered

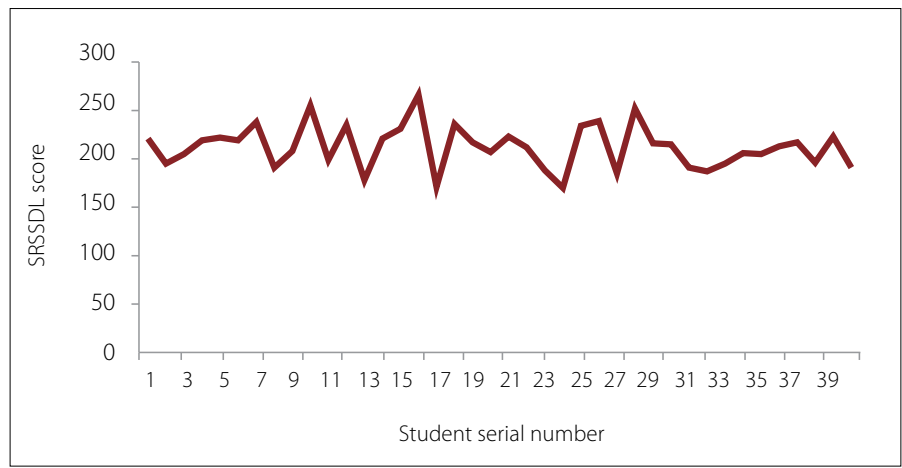

Fig. 1. The SRSSDL score against the student's serial number. Most of the students' scores are in the medium range (141 - 220), with no score in the low range (60 - 140).

Table 1. Summary of results of the qualitative aspect of the focus group discussion

\begin{tabular}{ll}
\hline Positive concepts & Negative concepts \\
\hline Self-motivated learning & Self-decided learning \\
Initiative & Self-teaching \\
Task shifting & \\
Interactive & \\
Partnership & \\
Guided learning &
\end{tabular}


the term SDL and discussed what it means for learning in the medical school. The categories identified were:

- Self-motivated learning. The perception of faculty was of a student who is enthusiastic about learning and is determined to learn. The following comment made during the focus group meeting illustrates this:

' $\ldots$ the main thing is that there is somebody who is trying to have some kind of self-motivation to learn something, hold it, improve on it without really being coerced, pushed, begged, there is self-determination to achieve something.' (Participant 3 )

- Initiative. Faculty embraced the idea of students showing some initiative in reading more than the scripted material:

'... we feel that there could be a room for initiative and also a room to explore other means even at the learning level; the students may be able to discover other things for themselves.' (Participant 7)

- Task shifting. This was seen as a positive aspect of SDL, as it transferred some responsibility for learning to the student and thus reduced the responsibility and workload of teachers. A statement of one of the participants conveys this:

'It [SDL] will reduce our [teachers'] work.' (Participant 8)

- Interactive. This conveyed the sense of being active, involved, talking to each other and changing each other. Interactive learning was seen to indicate learning in a group of which the teacher is a part, which was reflected by another teacher's concept of equality, and in which the traditional hierarchy between teacher and student was de-emphasised. Participants' comments on this in the focus group discussion were, inter alia, the following:

'... people in groups, making it learning, interacting ... ' (Participant 1)

'... sit, not in a classroom, in a circular form .... ' (Participant 1)

- Partnership. Faculty felt that success in the world sometimes hinged on partnerships and the same concept should be leveraged to help students feel more involved with their learning:

'... many of these companies in the United States succeeded because they made the staff part of the company.' (Participant 8 )

- Guided learning. The teachers seemed to emphasise the importance of guiding the students in the discovery of medical knowledge and to acquire relevant skills while following the prescribed curriculum:

'It's more of encouraging participation, that is what we are talking about, but still under, you know, a guide; still under the framework of an institution.' (Participant 4)

\section{Negative concepts}

After reviewing the definition of SDL as defined by Knowles, ${ }^{[1]}$ faculty members elicited some negative sentiment about it. The following negative categories were identified:

- Self-directed learning. Faculty were not keen on the idea that the students decide what and how to learn, as illustrated by the following quotes:

‘... if you put a medical student to stay on his own and start learning everything by himself, set goals for himself and decide appropriate learning strategies by himself, I don't think it's going to be better at the end of the day. He has a curriculum. He has a period of time he needs to learn. He needs to interact with others along the learning process.' (Participant 3)

'... for medical education, it will be difficult to allow the students to decide how they want to learn and what they want to learn before graduation if they all have to graduate within the same 6 or 7 years.' (Participant 5)

- Self-teaching (autodidactism). Learning by using the curriculum as a guide but without the teacher. This was expressed to question the trend of discussion that seemed to displace the teacher from the centre of the learning process:

'Giving us the impression the student is self-directed, doesn't need the teachers again.' (Participant 8 )

\section{Faculty's perception of the SRSSDL score of the students}

The faculty unanimously rated the self-directedness in learning of the finalyear medical students at the university as low. They expressed surprise that the students thought of themselves as having moderate SDL behaviour. This was a qualitative overview of the students' learning behaviour, as they did not go into the detail of reviewing the questionnaire and its various aspects.

'I would really want to score our student low ... but if we ... direct them in this self-directed learning focus, I guess from low they can get to high.' (Participant 1)

\section{Discussion}

Radical SDL is difficult to apply in formal education and is not an appropriate pedestal for teaching and learning in medical education - this summarises the view of the faculty leadership at the index medical school and is not new in the literature. ${ }^{[2,16]}$ This study adds the view of the Nigerian faculty about what SDL should entail in medical education, both in its positive and negative aspects, and the application of an SDL self-assessment tool to a group of Nigerian medical students. It also adds the results of applying the SRSSDL to a cohort of medical students, probably for the first time. Furthermore, it adds to previous research done in Africa and internationally. ${ }^{[10]}$

It is clear that the faculty perception in this study is in tandem with Schmidt ${ }^{[16]}$ with regard to the central role of teacher support for the selfmotivated student, but that there should be teacher guidance for those in need.

Scaffolding is an offshoot of the cognitive load theory of Sweller, in which the teacher provides support for the student in a learning task by providing a means of chunking the new information or skill, to more easily engage with working memory. ${ }^{[17,18]}$ The first contact with new information is through the sensory memory, but the individul becomes aware of the data when these move to the working memory, according to the cognitive load theory. ${ }^{[6]}$ Learning occurs when this information moves to the longterm memory, which has an infinite number of schematics to store and retrieve information. Cognitive overload occurs when the learner meets new information that is not organised into the schemata that allows movement to the long-term memory (usually about seven chunks). ${ }^{[6]}$ SDL needs to be staged according to the capacity of the learner by reducing the amount of scaffolding, as the learner matures in self-learning. ${ }^{[2]}$

Task-shifting from teacher to student would certainly make the teacher's work easier, but the focus should rather be on how it could enhance the 
student's learning. It seems that teachers do not always realise the benefits of self-discovery during the learning process and tend to want to be in charge of students' learning. However, the faculty in this study laud an interactive model of teaching and learning and, indeed, would be comfortable for students to partner with them in this process. This is reflected in the literature on PBL, which entrenches an interactive partnership model as a means of developing SDL in medical students..$^{[7]}$

From the results of this study, it appears that guided learning seems to be the core of the concept of how medical students can acquire the complex knowledge and skills required of a medical doctor. This is supported by the concept of cognitive load theory. Moreover, SRL is a teaching and learning method that utilises teacher guidance, which is more appropriate for the high cognitive load in medical education than SDL. Faculty was loath to adopt a concept that seemed to do away with the need for teachers. Rather, they preferred to shift more of the work of learning to the students in an interactive model, which makes students partners in the task of learning but under the guidance of teachers. In the PBL model of applying SDL, teachers are still necessary as facilitators. However, with the objective of developing SDL in medical students at the index school, faculty's concerns would have to be addressed.

The results of this study also demonstrate that there are some negative perceptions about the concept of SDL. Faculty members were concerned about the idea of SDL - this concept was expressed by the faculty as a facet of SDL that was not compatible with medical training, which has a defined curriculum and a fixed time of completion. Therefore, the view that a student can independently determine the subjects to study in a medical school (or any school), would be unusual to faculty in most medical schools and be precarious to the stakeholders to whom the school is socially accountable. ${ }^{[19]}$

Autodidaxy is a term that has been used synonymously with SDL in the literature; ${ }^{[20]}$ it speaks of self-learning or self-teaching and is considered a process in SDL. However, faculty members in this study seemed to be more concerned about autodidactism, which is self-teaching without formal education. ${ }^{[21]}$ Autodidactism is therefore more in tune with the comment of the faculty, who stated that giving the student the curriculum to study without the teachers would not be a reliable way to learn medicine.

The difficulty this faculty had with regard to defining SDL is common in the literature, more so with the need to safeguard the core aspects of the curriculum. ${ }^{[22]}$

Faculty was unanimous in rating the students in the low range of SDL behaviour and was rather surprised that the students' self-assessment placed them in the moderate range. This faculty rating can be misleading, as they have not been formally orientated to the workings of SDL, and so may not have the experience to make such a judgement. Rather, the general assessment by the faculty is more likely to represent their sense of the students' level of motivation, initiative and commitment to learning. It is also possible that the faculty have developed more SDL traits in the students than they credit themselves for.

This study may be the first where SRSSDL is being applied to medical students. Other studies have shown the application of the SDL readiness scale to medical students - somewhat different from SDL behaviour. ${ }^{[23]}$

The key findings of this study centre on the perceptions of the faculty focus group, which revealed a reluctance to participate in a venture that could make students decide what and when to learn. Rather, the group members had expectations about what it would take to produce self-directed learners and are willing to commit to this model, which can be paraphrased as follows: The self-motivated students demonstrating initiative under the guidance of teachers who use interactive forums for teaching. In addition, the teachers and students should partner towards the goal of ensuring that student learning takes place.

The modality for practically achieving this objective was not discussed, but the underlying ethos indicates a major need for training and orientation for both faculty and students.

This model appears to be similar to SRL in the following aspects: student motivation and initiative and teacher guidance in learning, suggesting that the faculty at the medical school would prefer to operate an SRL rather than an SDL framework for teaching. Nonetheless, SDL is a concept that is probably more relevant to a postgraduate medical doctor, who can use this approach to pursue lifelong learning and thus maintain safe, relevant and efficient practice for the duration of their professional life.

In view of the aim of medical schools, i.e. to produce self-directed, lifelong learners, there is a need to determine whether SRL would achieve this goal. Furthermore, it gives teachers more control and thus would seem more attuned to a hierarchical culture. The first author's (TEN) hierarchical cultural background may be reflected in the predominant teacher-centred culture of this medical school. Therefore, he may inadvertently prefer more teacher guidance in the teaching and learning framework in use at the index medical school. It would seem that changes in teaching and learning methods need to work with and leverage upon the background culture.

This work serves as a needs analysis with regard to the basic concepts of the faculty at this medical school to develop self-directed lifelong learners. Faculty development forums can therefore be designed that will be relevant to their needs.

The outcomes of this study are transferable to medical schools, especially in developing economies, which are yet to engage with producing selfdirected life-long learners.

\section{Study limitations}

To interpret the students' level of self-directedness in learning in detail would have required a faculty more conversant with the theories and practice of SDL. More depth of information on student behaviour, their challenges, and successes within the Nigerian context could have guided SDL implementation strategies.

The sampling of faculty was restricted to make the study 'doable', but also limited the conclusions from the study to those involved in the focus group. A systematic sampling of all faculty members would have yielded more valid results.

This study was conducted in a medical school that was yet to implement SDL; therefore, the definition of SDL may have been more difficult to grasp fully.

\section{Conclusion}

This study suggests that the final-year students have a low to moderate level of SDL behaviour. The index faculty are willing to develop teacher-guided self-motivated learning in their students, rather than strict SDL. Faculty should be concerned about this behaviour and should encourage SDL in students, in such a way that they realise its benefits to become lifelong learners. Further study on the perceptions about SRL are recommended. 
Author contributions. TEN conceived the study. AJNL participated in study conception. TEN collected the data and drafted the manuscript. AJNL participated in the drafting of the manuscript.

1. Knowles MS. Self-directed Learning - a Guide for Learners and Teachers. Chicago: Follett Publishing, 1975:18 2. Grow G. Teaching learners to be self-directed. Adult Educ Q 1991;41(3):125-149. http://dx.doi.org/10.1177/ 0001848191041003001

3. Loyens S, Magda J, Rikers R. Self-directed learning in problem-based learning and its relationships with selfregulated learning. Educ Psychol Rev 2008;20(4):411-427. http://dx.doi.org/10.1007/s10648-008-9082-7

4. Zimmerman BJ. Investigating self-regulation and motivation: Historical background, methodological developments, and future prospects. Am Educ Res J 2008;45(1):166. http://dx.doi.org/10.3102/0002831207312909

5. Kistner S, Rakoczy K, Otto B, Dignath-van Ewijk C, Büttner G, Klieme E. Promotion of self-regulated learning in classrooms: Investigating frequency, quality, and consequences for student performance. Metacogn Learn 2010;5(2):157-171. http://dx.doi.org/10.1007/s11409-010-9055-3

6. Young JQ, van Merrienboer J, Durning S, ten Cate O. Cognitive load theory: Implications for medical education: AMEE Guide No. 86. Med Teach 2014;36(5):371-384. http://dx.doi.org/10.3109/0142159X.2014.889290

7. Miflin BM, Campbell CB, Price DA. A conceptual framework to guide the development of self-directed, lifelong learning in problem-based medical curricula. Med Educ 2000;34(4):299-306. http://dx.doi.org/10.1046/j.13652923.2000.00564.x

8. Carrera LI, Tellez TE, D’Ottavio AE. Implementing a problem-based learning curriculum in an Argentinean medical school: Implications for developing countries. Acad Med 2003;78(8):798-801. http://dx.doi.org/10.1097/ 00001888-200308000-00010

9. Amin Z, Hoon Eng K, Gwee M, Dow Rhoon K, Chay Hoon T. Medical education in Southeast Asia: Emerging issues, challenges and opportunities. Med Educ 2005;39(8):829-832. http://dx.doi.org/10.1111/j.1365-2929.2005. $02229 x$
10. Greysen SR, Dovlo D, Olapade-Olaopa EO, Jacobs M, Sewankambo N, Mullan F. Medical education in sub-Sahara Africa: A literature review. Med Educ 2011;45(10):973-986. http://dx.doi.org/10.1111/j.1365-2923.2011.04039. 11. Olapade-Olaopa EO, ed. The 2010 MBBS Curriculum of the College of Medicine, University of Ibadan. Ibadan: College of Medicine, 2010

12. Gukas ID. Problem-based learning in undergraduate medical education: Can we really implement it in the Wes African subregion? West Afr J Med 2007;26(2):87-92

13. Olabiyi OO, Aiyegbusi AI, Noronha CC, Okanlawon AO. Students' view of a learning method: Opinions of firs year medical and dental students in the School of Basic Medical Sciences of University of Lagos, Nigeria, about problem based learning. Nig Q J Hosp Med 2008;18(4):185-190. http://dx.doi.org/10.4314/ngjhm.v18i4.45025

14. Creswell JW. Research Design: Qualitative, Quantitative, and Mixed Methods Approaches. 2nd ed. Thousand Oaks Sage, 2003:15-16.

5. Williamson SN. Development of a self-rating scale of self-directed learning. Nurse Res 2007;14(2):66-83. http:// dx.doi.org/10.7748/nr2007.01.14.2.66.c6022

16. Schmidt HG. Assumptions underlying self-directed learning may be false. Med Educ 2000;34(4):243-245. http:// doi.org/10.1046/j.1365-2923.2000.0656a.x

17. Jin J, Bridges SM. Educational technologies in problem-based learning in health sciences education: A systematic review. J Med Internet Res 2014;16(12):e251. http://dx.doi.org/10.2196/jmir.3240

18. Baker RM. Examples of scaffolding and chunking in online and blended learning environments, 2010. http:// dx.doi.org/10.2139/ssrn. 1608133

19. Boelen C, Woolard R. Social accountability: The extra leap to excellence for educational institutions. Med Teach 2011;33(8):614-619. http://dx.doi.org/10.3109/0142159X.2011.590248

. Ainoda N, Onishi H, Yasuda Y. Definitions and goals of 'self-directed learning' in contemporary medical education literature. Ann Acad Med Singapore 2005;34(8):515-519.

21. Wikipedia. Autodidacticism, 2014. http://en.wikipedia.org/wiki/Autodidacticism (accessed 5 January 2017).

22. Lunyk-Child OI, Crooks D, Ellis PJ, Ofosu C, O’Mara L, Rideout E. Self-directed learning: Faculty and studen perceptions. J Nurs Educ 2001;40(3):116-123.

23. Premkumar K, Pahwa P, Banerjee A, Baptiste K, Bhatt H, Lim HJ. Does medical training promote or deter self-directed learning? A longitudinal mixed-methods study. Acad Med 2013;88(11):1754-1764. http://dx dol org/10.1097/ACM.0b013e3182a9262d 